\title{
QUANTITATIVE $\mathrm{NH}_{2}$ CONCENTRATION DETERMINATION IN SHOCK TUBE LASER-ABSORPTION EXPERIMENTS
}

\author{
Katharina Kohse-Höinghaust, David F. Davidson, Albert Y. Chang, \\ and Ronald K. HaNsON \\ High Temperature Gasdynamics Laboratory, Department of Mechanical Engineering, \\ Stanford University, Stanford, CA 94305, U.S.A.
}

(Received 8 September 1988)

\begin{abstract}
The absorption coefficient of the $\tilde{A}^{2} A_{1} \leftarrow \tilde{X}^{2} B_{1}(090 \leftarrow 000) \Sigma^{P} Q_{1, N} 7$ transition in $\mathbf{N H}_{2}$ at $16,739.90 \mathrm{~cm}^{-1}$ has been measured in a shock tube as a function of temperature using a cw narrowband absorption laser. For controlled $\mathbf{N H}_{2}$ generation, both $\mathbf{N H}_{3}$ photolysis at $193 \mathrm{~nm}$ and $\mathrm{NH}_{3}$ pyrolysis were used; both calibration methods are independent of thermochemical data. Rapid scanning of the laser in pyrolysis and laser-photolysis experiments provided spectroscopic data for the splitting and the intensity ratio of the two spin components of the doublet transition, as well as information on collisional broadening by argon. From these experiments, the oscillator strength of the transition was inferred to be $(6.35 \pm 1.90) \times 10^{-5}$. The shock tube diagnostic method for $\mathrm{NH}_{2}$ developed here is well-suited for a variety of reaction kinetics experiments.
\end{abstract}

\section{INTRODUCTION}

The $\mathrm{NH}_{2}$ radical is an important intermediate in nitrogen-containing combustion systems. It is one of the $\mathrm{NH}_{i}$ species which control the branching to $\mathrm{NO}$ or $\mathrm{N}_{2}$ formation in fuel-nitrogen conversion, ${ }^{1}$ and its key role in the Thermal DeNO process for NO removal has been pointed out in several studies. ${ }^{2-4}$ High temperature investigations of the detailed kinetics of the radical, using directly monitored absolute $\mathrm{NH}_{2}$ concentrations, could contribute to the understanding of these complex reaction systems.

$\mathrm{NH}_{2}$ has been detected in flames ${ }^{5-9}$ and flow cells ${ }^{10-12}$ using laser-induced fluorescence ${ }^{5,9-11}$ and laser-absorption ${ }^{6-8,12}$ techniques. Sources, detection, and elementary kinetics of $\mathrm{NH}_{2}$ have been reviewed recently by Lesclaux. ${ }^{13}$ For high-temperature kinetics studies in a shock tube, $\mathrm{NH}_{2}$ has previously been monitored by its visible emission. ${ }^{14,15}$

Recently, laser-absorption techniques were introduced as a valuable tool for the detection of molecular radicals in a shock tube. ${ }^{16}$ In the present study, we extend this method to $\mathrm{NH}_{2}$. Whereas line and band-oscillator strengths required for the quantitative calculation of absorption coefficients are well-known for most diatomic radicals of interest, we encountered a different situation for $\mathrm{NH}_{2}$. Chou et $\mathrm{al}^{7}$ determined the oscillator strength for the $\mathrm{NH}_{2} \tilde{A}^{2} A_{1} \leftarrow \bar{X}^{2} B_{1}(090 \leftarrow 000) \Sigma p Q_{1, N} 7$ transition from laser-absorption measurements in a flame under partial equilibrium conditions. Later, the same group reported ${ }^{8}$ an oscillator strength which was obtained from an extended set of data in the same flames with a slightly different method and which was about a factor of 3 lower. Both methods of generating known $\mathrm{NH}_{2}$ concentrations involved the heats of formation of $\mathrm{NH}$ or $\mathrm{NH}_{2}$, so that the reported oscillator strengths depend in a sensitive way on the thermodynamic data used in their determination. Although a theoretical calculation ${ }^{9}$ of the oscillator strength for the same line seemed to support the earlier ${ }^{7}$ experimental result, a reevaluation ${ }^{17}$ of the experimental oscillator strengths from the flame experiments of Refs. 7 and 8 with currently accepted heats of formation resulted in disagreement between experimental and theoretical values by about a factor of 4 . It must be concluded that, at present, oscillator strengths for $\mathrm{NH}_{2}$ are not known well enough to enable accurate calculation of the line-center absorption coefficient and, hence, quantitative detection of $\mathrm{NH}_{2}$ by narrow-line laser absorption.

tOn leave from DFVLR, Institut für Physikalische Chemie der Verbrennung, D-7000 Stuttgart, F.R.G. 
We therefore designed shock-tube experiments which provide us with a controlled source of $\mathrm{NH}_{2}$ for a direct measurement of the $\mathrm{NH}_{2}$ absorption coefficient. These experiments produced $\mathrm{NH}_{2}$ both by $\mathrm{ArF}$ excimer laser photolysis of $\mathrm{NH}_{3}$ and by direct shock-tube pyrolysis in $\mathrm{NH}_{3} / \mathrm{Ar}$ mixtures.

\section{EXPERIMENT}

The experimental arrangement consists of the shock tube itself, a narrow-bandwidth $\mathrm{cw}$ laser absorption system and a photolysis laser. Some details of the apparatus have been reported elsewhere. ${ }^{15,16,18}$

The experiments were carried out behind reflected shock waves, using either a conventional pyrolysis set-up or one of two separate arrangements for laser photolysis; the photolysis laser beam was aligned either collinearly with the probe-laser beam or, by passing the photolysis beam through the shock tube end-wall, at right angles to the probe laser beam. Although most experiments were carried out with a fixed laser frequency, we additionally used a previously established, ${ }^{19}$ rapid frequency-scanning technique; these experiments were performed both in the pyrolysis and in the end-wall photolysis arrangement. Figure 1 gives some important details of the apparatus, shown here for the end-wall photolysis configuration.

The stainless-steel shock tube has a $14.3 \mathrm{~cm}$ dia driven section of $6 \mathrm{~m}$ length and a driver section of $5 \mathrm{~cm}$ dia and $2.4 \mathrm{~m}$ length. Helium was used for the driver gas. Temperatures and pressures in the reflected shock regime were calculated from the initial pressures and temperatures and the shock speeds which were monitored with a series of four thin-film gauges mounted along the tube. Post-shock pressures were independently verified with a Kistler model 603B1 piezo-electric transducer. Mixtures of $\sim 0.1-2 \% \mathrm{NH}_{3}$ in $\mathrm{Ar}$ by volume were prepared with a diffusion-pumped gas-handling manifold; gas mixtures for about 10 consecutive shocks were stored in a 221 . stainless-steel, stirred mixing tank. To ensure reproducibility in $\mathrm{NH}_{3}$ concentration, the shock tube was passivated with the mixture for $5 \mathrm{~min}$ and then diffusion-pumped for $5 \mathrm{~min}$ prior to filling the tube just before each shock. This passivation procedure was validated by detection of the $\mathrm{NH}_{3}$ infrared (i.r.) emission with an Judson IR InSb detector with a 2-3.4 $\mu \mathrm{m}$ bandpass filter. The residual uncertainty in the initial $\mathbf{N H}_{3}$ mole fraction was found to be $<5 \%$.

In the fixed-frequency experiments, $\mathrm{NH}_{2}$ was detected by absorption in the $\tilde{A}^{2} A_{1} \leftarrow \tilde{X}^{2} B_{1}(090 \leftarrow 000) \Sigma$ sub-band on the isolated ${ }^{P} Q_{1, N} 7$ transition at $16,739.90 \mathrm{~cm}^{-1}$. A SpectraPhysics Model 380 ring dye laser (pumped with 3-4 W all lines from a Spectra-Physics Model $171 \mathrm{Ar}^{+}$laser) was used with rhodamine $6 \mathrm{G}$ dye. The single-mode quality of the output beam was verified with an Spectra-Physics model $4708 \mathrm{GHz}$ scanning interferometer; the output wavelength was monitored with a Burleigh WA-10 wavemeter. The dye laser power output was typically 30-35 $\mathrm{mW}$. The dye laser beam was focussed into the shock tube through a pair of either Suprasil or $\mathrm{CaF}_{2}$ windows with a beam diameter of about $1.5 \mathrm{~mm}$. The windows were located $21 \mathrm{~mm}$ from the shock tube end-wall for most of the experiments; this distance was $9.5 \mathrm{~mm}$ in the end-wall photolysis arrangement. Before entering the shock tube, a portion of the absorption laser beam

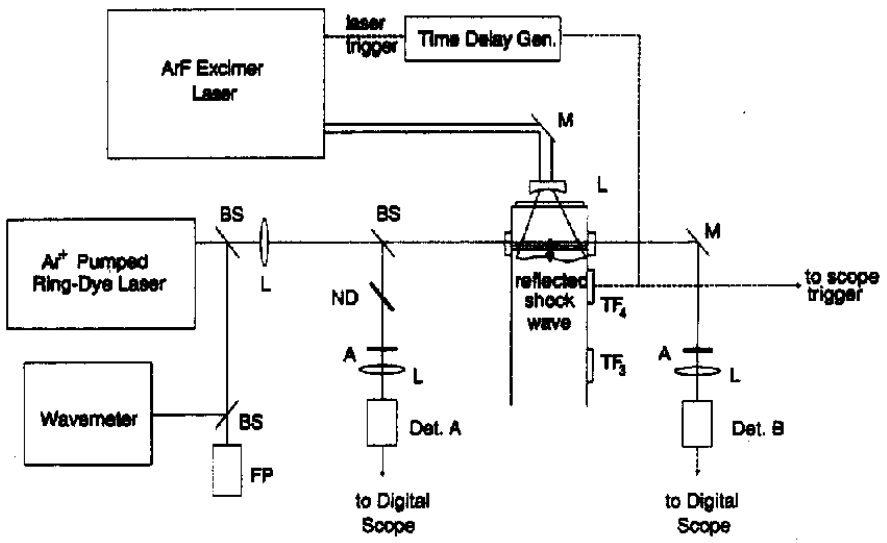

Fig. 1. Experimental arrangement for end-wall photolysis. A-Aperture; BS-beam splitter; Det. A, B-photodiodes; FP-Fabry-Perot etalon; L-lens; M-mirror; ND-neutral density filter; TF-thinfilm gauge. 

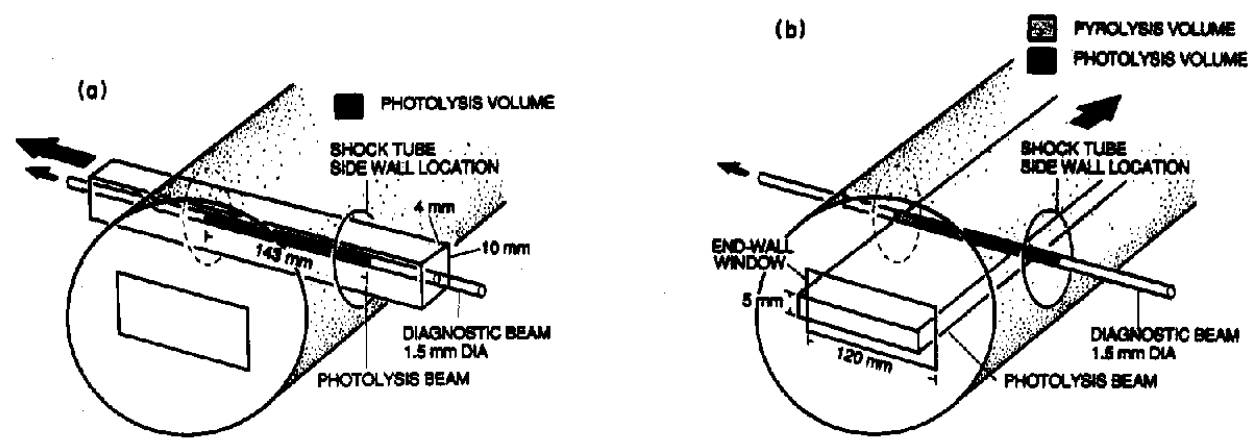

Fig. 2. Interaction volume of the excimer laser and the absorption laser beams in (a) collinear and (b) end-wall photolysis experiments.

was split off to provide a reference beam. Reference and probe beam intensities were balanced with neutral density filters and monitored with EG\&G UV100B silicon photodiodes and matched amplifiers. The beams were focussed onto the detectors so that the beam diameters were about half the diameter of the active surface of the photodiodes. Probe and reference signals, as well as the a.c.-coupled difference signal, were recorded and stored on Nicolet Model 2090 digital oscilloscopes (sampling time $0.5 \mu \mathrm{sec}$ ). The oscilloscopes were triggered from the thin-film gauge closest to the end wall. Typically, the probe and reference signals of $2-6 \mathrm{~V}$ exhibited about $1 \%$ amplitude fluctuations; the residual fluctuations on the difference signal were $0.5-5 \mathrm{mV}$. The minimum useful absorption signal was on the order of $0.1 \%(\mathrm{~S} / \mathrm{N} \geqslant 3)$, depending on the particular conditions.

The Questek Model 2220 ArF excimer laser used for the photolysis experiments produced $40-150 \mathrm{~mJ}$ at $193 \mathrm{~nm}$ and had a spectral bandwidth of $0.5 \mathrm{~nm}$. In the collinear photolysis experiments, the unfocussed excimer laser radiation entered the shock tube with an incident power density of $5-17 \mathrm{~mJ} / \mathrm{cm}^{2}$. The spatial beam profile was flat to within $10 \%$ across a $10 \mathrm{~mm}$ vertical by $4 \mathrm{~mm}$ horizontal area. Figure 2(a) shows the laser beams for this geometry. After passing through the shock tube, the excimer radiation was blocked from the absorption beam path with a Schott WG 280 filter. In some of the experiments, the filter was replaced by a mirror to allow a second pass of both beams through the shock tube. An Abtronics Model 200 variable time delay generator was used to delay the excimer pulse by $20-100 \mu \mathrm{sec}$ with respect to the arrival of the reflected shock front at the observation volume.

For the end-wall photolysis experiments, the ArF excimer laser beam was expanded with an $f=-300 \mathrm{~mm}$ cylindrical lens to a rectangle of $13 \mathrm{~cm} \times 5 \mathrm{~mm}$. This configuration is shown in Fig. 2(b). The excimer beam fully irradiated the length of a $12.1 \mathrm{~cm}$ Suprasil window mounted in the shock tube end-wall. The spatial profile of the excimer laser power was measured to be flat within $10 \%$ with an incident power density of $2-7 \mathrm{~mJ} / \mathrm{cm}^{2}$. The two laser beams were carefully aligned to ensure full overlap in the shock tube.

In the rapid-scanning experiments, the laser frequency was swept across the doublet line of interest by means of a dual-rhomb tuner inside the cavity. This type of measurement was performed following Ref. 19, where a similar experiment is described in detail for the $\mathrm{OH}$ radical. In these experiments, the $8 \mathrm{GHz}$ scanning interferometer was replaced with a fixed $(2.00 \pm 0.005 \mathrm{GHz})$ etalon. The optical balancing of the probe and reference signals was found to be more difficult than in the fixed frequency experiments, probably due to etalon effects resulting from the frequency modulation of the laser. Thus a different beam balancing strategy was used. The probe and reference beams were sampled using front-surface partial reflections off of $5 \mathrm{~mm}$ thick fused silica plates placed before and after the shock tube. The beam intensities were matched by varying the angles of these plates, thus changing the reflection coefficient. This seemed to improve the noise characteristics of the signal.

\section{RESULTS AND DISCUSSION}

\subsection{Absorption coefficient}

Before the absorption coefficient was determined, the absorption spectrum in various shockheated $\mathrm{NH}_{3} / \mathrm{Ar}$ mixtures was verified to be consistent with the known $\mathrm{NH}_{2}$ spectrum. This was done 
by repeating a series of shocks at the same conditions, while stepwise changing the fixed frequency of the laser between shocks. Agreement with tabulated line positions ${ }^{20}$ was found in the spectral range investigated (the ${ }^{P} Q_{1, N} \Sigma$ sub-band).

Two different experimental schemes, based on photolysis and pyrolysis, were used for generating the known $\mathrm{NH}_{2}$ concentrations needed for determination of the $\mathbf{N H}_{2}$ absorption coefficient.

3.1.1. $\mathrm{NH}_{3}$ photolysis experiments. In the $\mathrm{UV}$ and VUV, the $\mathrm{NH}_{3}$ spectrum exhibits a sequence of broad vibrational structures ${ }^{21}$ with individual lobes significantly broader than the bandwidth of our photolysis laser. No rotational structure has been observed. The $\mathrm{NH}_{3}$ spectrum can thus be regarded to good approximation as a continuum over the bandwidth of the photolysis laser. The photolysis of $\mathrm{NH}_{3}$ with $193 \mathrm{~nm}$ produces $\mathrm{NH}_{2}$ and $\mathrm{H}$ with a quantum yield near unity. ${ }^{12} \mathrm{NH}_{2}$ is generated predominantly in its ground electronic state. ${ }^{21,22}$ About $2.5 \%$ of the $\mathrm{NH}_{2}$ radicals are formed in the excited $\tilde{A}^{2} A_{1}$ state, ${ }^{22}$ which is then rapidly quenched to the ground state. ${ }^{13,23} \mathrm{NH}$ is formed by single-photon dissocation in much smaller quantities. ${ }^{24}$ Recent investigations ${ }^{25,26}$ review a number of earlier studies and provide a fairly complete understanding of the photodissociation mechanisms. It has been pointed out ${ }^{26}$ that the ground state $\mathrm{NH}_{2}$ fragment in the $\mathrm{ArF}$ excimer photolysis is initially formed with high internal energy, preferentially with significant rotation about the $a$-axis. However, for the near atmospheric pressure and the $>1 \mu \mathrm{sec}$ time scale of our experiments, the $\mathrm{NH}_{2}$ energy distribution can be assumed to be close to thermal as potential non-thermal distributions of the nascent $\mathrm{NH}_{2}$ are relaxed rapidly. ${ }^{22,25}$ Likewise any spatial-orientation preference of the $\mathrm{NH}_{2}$ molecule is quickly destroyed.

The $\mathrm{NH}_{2}$ absorption can be described by Beer's law as

$$
\ln \left(I_{0} / I\right)=k_{\mathrm{NH}_{2}} p \chi_{\mathrm{NH}_{2}} L
$$

where $I_{0}$ and $I$ are the intensities of the reference and the probe beams, respectively, $k_{\mathrm{NH}_{2}}$ $\left(\mathrm{cm}^{-1} \mathrm{~atm}^{-1}\right)$ is the linecenter absorption coefficient of the $(090 \leftarrow 000) \Sigma^{P} Q_{1, N} 7$ line at temperature $T(\mathrm{~K}), p(\mathrm{~atm})$ is the reflected shock pressure, $\chi_{\mathrm{NH}_{2}}$ is the $\mathrm{NH}_{2}$ mole fraction, and $L(\mathrm{~cm})$ the absorption path length.

The $\mathrm{NH}_{2}$ mole fraction created by photolysis is calculated following the description in Ref. 18 . Each absorbed $193 \mathrm{~nm}$ photon changes one $\mathrm{NH}_{3}$ molecule into one $\mathrm{NH}_{2}$ and one $\mathrm{H}$ molecule. For the optically thin case of $193 \mathrm{~nm}$ absorption, the $\mathrm{NH}_{2}$ concentration is obtained simply from

$$
\chi_{\mathrm{NH}_{2}}=\chi_{\mathrm{NH}_{2}} I_{0,193} k_{193} p / N_{\text {tot }},
$$

where $k_{193}\left(\mathrm{~cm}^{-1} \mathrm{~atm}^{-1}\right)$ is the $\mathrm{NH}_{3}$ absorption coefficient, $N_{\text {tor }}\left(\mathrm{cm}^{-3}\right)$ the total species number density, and $I_{0,193}$ the number $/ \mathrm{cm}^{3}$ of incident $193 \mathrm{~nm}$ photons. For the optically-thick case, the analysis is more complicated as the successive parts of incident photon pulse see a diminishing $\mathrm{NH}_{3}$ concentration caused by the absorption of the leading parts of the pulse. The resulting calculation follows the same principle as that of Eq. (2), and is described in detail in Ref. 18.

For the collinear case and large $193 \mathrm{~nm}$ optical depth, the resulting $\mathrm{NH}_{2}$ profile is necessarily inhomogeneous along $L$. The end-wall photolysis experiments offer the advantage that the number of incident photons per $\mathrm{cm}^{2}$ is equal along the entire probe laser absorption path length, resulting in an homogeneous mole fraction of $\mathrm{NH}_{2}$ along $L$. A disadvantage of the end-wall arrangement is that the size of the end-wall window does not permit the irradiation of the entire horizontal cross section of the shock tube. Thus, if photolysis rather than pyrolysis is the dominant $\mathrm{NH}_{2}$-generating process, the effective path length for $\mathrm{NH}_{2}$ absorption is taken to be the horizontal dimension of the end-wall window $\left(L=12.1 \mathrm{~cm}\right.$ ). In cases of significant pyrolysis combined with additional $\mathrm{NH}_{2}$ generation by photolysis, the different path lengths for photolysis and pyrolysis creation of $\mathrm{NH}_{2}$ is accounted for in the evaluation of the absorption signals.

To complete the calculation of the $\mathrm{NH}_{2}$ mole fraction in Eq. (2), the absorption coefficient of $193 \mathrm{~nm}$ radiation by $\mathrm{NH}_{3}$ is needed. $k_{193}$ was measured in the shock tube as a function of temperature in mixtures of typically $0.4 \% \mathrm{NH}_{3}$ in Ar using different laser energies in the range from room temperature to $3000 \mathrm{~K}$. The details of the experiment will be reported in Ref. 27. Figure 3 shows the measured $\mathrm{NH}_{3}$ absorption coefficient at $193 \mathrm{~nm}$ as a function of temperature. At room temperature, the absorption coefficient from this experiment agrees within $20 \%$ with literature data. ${ }^{24,28,29}$ Absorption of one $193 \mathrm{~nm}$ photon excites $\mathrm{NH}_{3}$ from the $\hat{X}^{\prime} A_{1} v_{2}^{\prime \prime}=0$ level to the predissociative $\tilde{A}^{1} A_{2}^{\prime \prime} v_{2}^{\prime}=6$ state. ${ }^{22,25}$ The temperature dependence of the absorption coefficient 


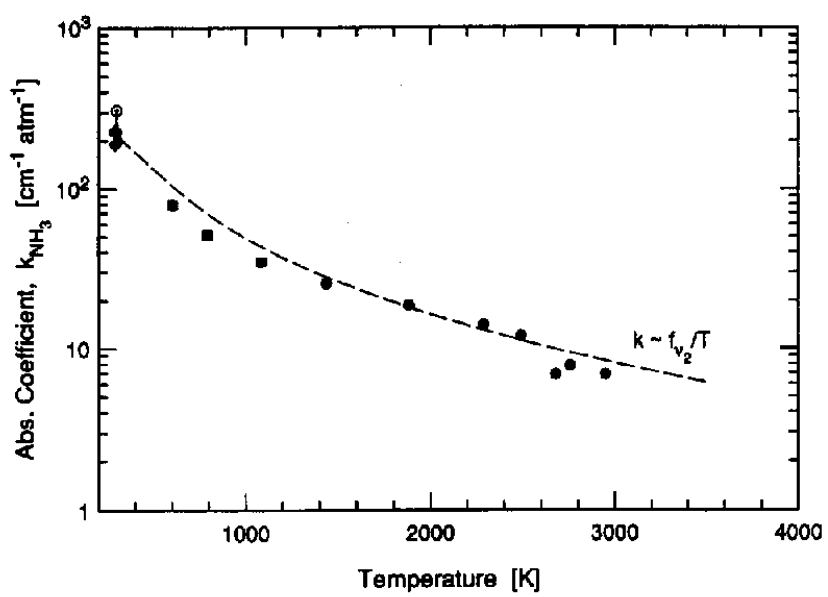

Fig. 3. Absorption coefficient of $\mathrm{NH}_{3}$ at the ArF laser wavelength vs temperature. ( $\$$ ), shock tube used as a static cell; ( $\square$ ), incident shock data; (O), reflected shock data; (O), Ref. 24; (bar), interpolated from graphs in Refs. 28 and 29 . The dashed line shows the expected temperature dependence as a function of the density $(1 / T)$ and the population in the lower level for the absorbing transition $\left(f_{v_{2}}\right)$.

appears to vary as the temperature-dependent population $\left(f_{v 2}\right)$ in the $\widetilde{X}^{1} A_{1} v_{2}^{\prime \prime}=0$ level scaled by the variation in density $\left(T^{-1}\right)$ as shown by the dashed line in Fig. 3.

In the $\mathrm{NH}_{2}$ absorption experiments, the incident excimer laser intensity $I_{0,193}$ is determined from a measured calibration factor which relates the displayed single-pulse energy readout (in $\mathrm{mJ}$ ) of the excimer laser to the incident photon density. These calibration factors are obtained from measurements with a power meter at the centerline of the excimer beam in the shock tube for the two geometries, using slits of different known areas. This center region of the beam corresponds in both geometries to the flat part of the spatial profile, in which the ring dye laser beam for the $\mathbf{N H}_{2}$ diagnostic overlaps the excimer laser beam. The estimated uncertainty in the measurement of $I_{0,193}$ is $20 \%$.

With the initial mole fraction of $\mathrm{NH}_{2}$ evaluated from Eq. (2), the measured absorption trace is fitted with a kinetic model to extract the $\mathrm{NH}_{2}$ absorption coefficient. This is necessary under our conditions, as the $\mathrm{H}$ atoms generated in the photolysis rapidly react to form additional $\mathrm{NH}_{2}$ by the reaction $\mathrm{NH}_{3}+\mathrm{H} \rightarrow \mathrm{NH}_{2}+\mathrm{H}_{2}$. It would thus be misleading to assume that all $\mathrm{NH}_{2}$ absorption in the experiment, even at early times, originates from the photolysis alone. Depending on the concentration of $\mathrm{NH}_{3}$ and the number of incident excimer laser photons, the $\mathrm{NH}_{2}$ additionally created by this reaction can increase the total $\mathrm{NH}_{2}$ concentration by as much as $\sim 70 \%$. This is therefore taken into account in the determination of the $\mathrm{NH}_{2}$ absorption coefficient. Using the chemical kinetic software package CHEMKIN ${ }^{30}$ with the reaction set given in Table 1 and the initial $\mathrm{NH}_{2}$ (and $\mathrm{H}$ ) mole fraction calculated from Eq. (2), or, in the general case, following the analysis of Ref. 18 , the experimental $\mathrm{NH}_{2}$ profiles are scaled to match those calculated with the model by varying only the assumed $\mathrm{NH}_{2}$ absorption coefficient. The matching of the experimental and simulated concentrations is performed with particular emphasis on early times, where the $\mathrm{NH}_{2}$ profile is sensitive only to the reaction of $\mathrm{NH}_{3}$ with $\mathrm{H}$ (reaction 23, Table 1) and, to a lesser extent, to the reaction of $\mathrm{NH}_{2}$ with $\mathrm{H}$ (reaction 14). The rate coefficient for reaction 23 has recently been measured by different groups, ${ }^{31,32,33}$ with very good agreement obtained over a large temperature range. We use the expression given by Michael et $a^{32}$ over the entire temperature range from 1600 to $3200 \mathrm{~K}$. Though the amount of $\mathrm{NH}_{2}$ formed by this reaction depends crucially on the value chosen for $k_{23}$, this does not imply a large uncertainty in the $\mathrm{NH}_{2}$ absorption coefficient. Even in the limit that we ignore the formation of additional $\mathrm{NH}_{2}$ by this reaction entirely, the inferred absorption coefficient would be only $30-40 \%$ higher. A variation of $k_{23}$ by $\pm 25 \%$ (which is within the range of the uncertainty attributable to this reaction coefficient) changes the absorption coefficient by $<10 \%$. Similarly, a variation of $k_{14}$ by a factor of 2 (the expected uncertainty) results in an uncertainty in the absorption coefficient of about $10 \%$. 
Table 1. Reaction mechanism for the $\mathrm{NH}_{2}$ calibration experiments; $k=A T^{\mathrm{B}} \exp -(E / R T), R=1.987 \mathrm{cal} \mathrm{K}^{-1} \mathrm{~mol}^{-1}, k$ in $\mathrm{cm}^{3} \mathrm{~mol}^{-1} \mathrm{sec}^{-1}$.

\begin{tabular}{|c|c|c|c|c|c|c|}
\hline \multicolumn{2}{|c|}{ Number } & Reaction & A & B & E & Ref. \\
\hline \multirow{9}{*}{$t$} & 1 & $\mathrm{H}+\mathrm{H}+\mathrm{M}=\mathrm{H}_{2}+\mathrm{M}$ & $1.00 \mathrm{E} 18$ & -1.0 & 0 & 51 \\
\hline & 2 & $\mathrm{H}+\mathrm{H}+\mathrm{H}_{2}=\mathrm{H}_{2}+\mathrm{H}_{2}$ & 9.20 E 16 & -0.6 & 0 & 51 \\
\hline & 3 & $N_{2}+M=N+N+M$ & 7.41 E 20 & -1.6 & 224,825 & 52 \\
\hline & 4 & $N H+M=N+H+M$ & $2.50 \mathrm{E} 15$ & 0.0 & 83,416 & 53 \\
\hline & 5 & $\mathrm{NH}_{2}+\mathrm{M}-\mathrm{NH}+\mathrm{H}+\mathrm{M}$ & 3.16 E 23 & -2.0 & 91,360 & 52 \\
\hline & 6 & $\mathrm{~N}_{2} \mathrm{H}+\mathrm{M}=\mathrm{N}_{2}+\mathrm{H}+\mathrm{M}$ & $2.00 \mathrm{E} 14$ & 0.0 & 19,861 & 52 \\
\hline & 7 & $\mathrm{~N}_{2} \mathrm{H}=\mathrm{N}_{2}+\mathrm{H}$ & $1.00 \mathrm{E} \mathrm{O4}$ & 0.0 & 0 & 34 \\
\hline & B & $\mathrm{NH}+\mathrm{H}=\mathrm{N}+\mathrm{H}_{2}$ & 1.00 E 14 & 0.0 & 0 & 34 \\
\hline & 8 & $\mathrm{NH}+\mathrm{N}=\mathrm{N}_{2}+\mathrm{H}$ & $3.00 \mathrm{E} 13$ & 0.0 & 0 & 34 \\
\hline \multirow[t]{4}{*}{$\neq$} & 10 & $\mathrm{NH}_{3}+\mathrm{M}=\mathrm{NH}_{2}+\mathrm{H}+\mathrm{M}$ & $2.51 E 16$ & 0.0 & 93,743 & 52 \\
\hline & 11 & $\mathrm{NH}_{3}+\mathrm{M}=\mathrm{NH}+\mathrm{H}_{2}+\mathrm{M}$ & 6.31 E 14 & 0.0 & 93,346 & 52 \\
\hline & 12 & $\mathrm{~N}_{2} \mathrm{H}_{2}+\mathrm{M}=\mathrm{N}_{2} \mathrm{H}+\mathrm{H}+\mathrm{M}$ & 5.00 E 16 & 0.0 & 50,000 & 1 \\
\hline & 13 & $\mathrm{~N}_{2} \mathrm{H}_{2}+\mathrm{M}=\mathrm{NH}+\mathrm{NH}+\mathrm{M}$ & 3.16 E 16 & 0.0 & 99,300 & 52 \\
\hline \multirow[t]{3}{*}{$\neq$} & 14 & $\mathrm{NH}_{2}+\mathrm{H}=\mathrm{NH}+\mathrm{H}_{2}$ & 6.92 E 13 & 0.0 & 3,650 & 54 \\
\hline & 15 & $\mathrm{NH}+\mathrm{NH}=\mathrm{N}_{2}+\mathrm{H}+\mathrm{H}$ & $4.00 \mathrm{E} 13$ & 0.0 & 0 & 53 \\
\hline & 16 & $\mathrm{~N}_{2} \mathrm{H}+\mathrm{H}-\mathrm{N}_{2}+\mathrm{H}_{2}$ & $1.00 \mathrm{E} 14$ & 0.0 & 0 & 34 \\
\hline \multirow[t]{5}{*}{+} & 17 & $\mathrm{~N}+\mathrm{NH}_{2}=\mathrm{N}_{2}+\mathrm{H}+\mathrm{H}$ & 7.20 E 13 & 0.0 & 0 & 34 \\
\hline & $1 B$ & $\mathrm{~N}_{2} \mathrm{H}+\mathrm{N}=\mathrm{NH}+\mathrm{N}_{2}$ & 3.16 E 13 & 0.0 & 1,986 & 52 \\
\hline & 19 & $\mathrm{~N}_{2} \mathrm{H}_{3}+\mathrm{M}-\mathrm{N}_{2} \mathrm{H}_{2}+\mathrm{H}+\mathrm{M}$ & $1.00 \mathrm{E} 16$ & 0.0 & 49,652 & 52 \\
\hline & 20 & $\mathrm{~N}_{2} \mathrm{H}_{3}+\mathrm{M}=\mathrm{NH}_{2}+\mathrm{NH}+\mathrm{M}$ & $1.00 \mathrm{E} 16$ & 0.0 & $41,70 B$ & 52 \\
\hline & 21 & $\mathrm{~N}_{2} \mathrm{H}_{2}+\mathrm{H}=\mathrm{N}_{2} \mathrm{H}+\mathrm{H}_{2}$ & 5.00 E 13 & 0.0 & 1,000 & 1 \\
\hline$\uparrow$ & 22 & $\mathrm{NH}+\mathrm{NH}_{2}=\mathrm{NH}_{3}+\mathrm{N}$ & $1.00 \mathrm{E} 13$ & 0.0 & 0 & 53 \\
\hline$\neq$ & 23 & $\mathrm{NH}_{3}+\mathrm{H}=\mathrm{NH}_{2}+\mathrm{H}_{2}$ & 6.36 E 05 & 2.39 & 10,171 & 32 \\
\hline \multirow[t]{6}{*}{$\neq$} & 24 & $\mathrm{NH}+\mathrm{NH}_{2}=\mathrm{N}_{2} \mathrm{H}_{2}+\mathrm{H}$ & $5.00 \mathrm{E} 13$ & 0.0 & 0 & 1 \\
\hline & 25 & $\mathrm{~N}_{2} \mathrm{H}+\mathrm{NH}=\mathrm{N}_{2}+\mathrm{NH}_{2}$ & $5.00 \mathrm{E} 13$ & 0.0 & 0 & 34 \\
\hline & 26 & $\mathrm{~N}_{2} \mathrm{H}_{3}+\mathrm{H}=\mathrm{N}_{2} \mathrm{H}_{2}+\mathrm{H}_{2}$ & 1.00E 12 & 0.0 & 1,986 & 52 \\
\hline & 27 & $\mathrm{~N}_{2} \mathrm{H}_{3}+\mathrm{H}=\mathrm{NH}_{2}+\mathrm{NH}_{2}$ & $1.58 \mathrm{E} 12$ & 0.0 & 0 & 52 \\
\hline & 28 & $\mathrm{~N}_{2} \mathrm{H}_{3}+\mathrm{H}=\mathrm{NH}+\mathrm{NH}_{3}$ & $1.00 \mathrm{E} 11$ & 0.0 & 0 & 52 \\
\hline & 29 & $\mathrm{~N}_{2} \mathrm{H}+\mathrm{NH}_{2}=\mathrm{N}_{2}+\mathrm{NH}_{3}$ & 5.00 E 13 & 0.0 & 0 & 1 \\
\hline \multirow[t]{4}{*}{+} & 30 & $\mathrm{NH}_{2}+\mathrm{NH}_{2}=\mathrm{N}_{2} \mathrm{H}_{2}+\mathrm{H}_{2}$ & $5.00 \mathrm{E} 11$ & 0.0 & 0 & 1 \\
\hline & 31 & $\mathrm{~N}_{2} \mathrm{H}_{2}+\mathrm{NH}=\mathrm{N}_{2} \mathrm{H}+\mathrm{NH}_{2}$ & $1.00 \mathrm{E} 13$ & 0.0 & 1,000 & 1 \\
\hline & 32 & $\mathrm{NH}_{3}+\mathrm{NH}_{2}-\mathrm{N}_{2} \mathrm{H}_{3}+\mathrm{H}_{2}$ & 7.94 E 11 & 0.0 & 21,549 & 52 \\
\hline & 33 & $\mathrm{~N}_{2} \mathrm{H}_{2}+\mathrm{NH}_{2}=\mathrm{NH}_{3}+\mathrm{N}_{2} \mathrm{H}$ & 1.00 E 13 & 0.0 & 1,000 & 1 \\
\hline
\end{tabular}

+Important reactions under certain conditions.

†Important reactions.

Figure 4 gives an example of the determination of the $\mathrm{NH}_{2}$ absorption coefficient from an end-wall photolysis experiment. The time axis is chosen such that the excimer pulse occurs at $t_{0}=0$. The uncertainty in $t_{0}$ is negligible, as the ArF laser pulse duration of $\sim 25 \mathrm{n} \mathrm{sec}$ is very short compared to the time scale of Fig. 4. No pyrolysis formation of $\mathrm{NH}_{2}$ is observed at this temperature of $1780 \mathrm{~K}$ (at higher temperatures pyrolysis can occur prior to the photolysis pulse). The sharp rise in concentration at $t_{0}=0$ corresponds to the photolysis, which in this case generates $60 \mathrm{ppm} \mathrm{NH}_{2}$. The early part of the $\mathrm{NH}_{2}$ profile is best approximated with an absorption coefficient of $5.2 \mathrm{~cm}^{-1} \mathrm{~atm}^{-1}$.

Consistent results were obtained from a total of about 40 photolysis experiments, two-fifths of them in the collinear geometry. The $\mathrm{NH}_{3}$ concentration as well as the laser energy were varied by factors of 10 in the temperature range of $1600-2200 \mathrm{~K}$, corresponding to initial $\mathrm{NH}_{2}$ mole fractions of $20-200 \mathrm{ppm}$. The resulting pressures were in the range of $0.9-1.2 \mathrm{~atm}$, however for near atmospheric pressures the results are expected to be only weakly dependent on pressure. 


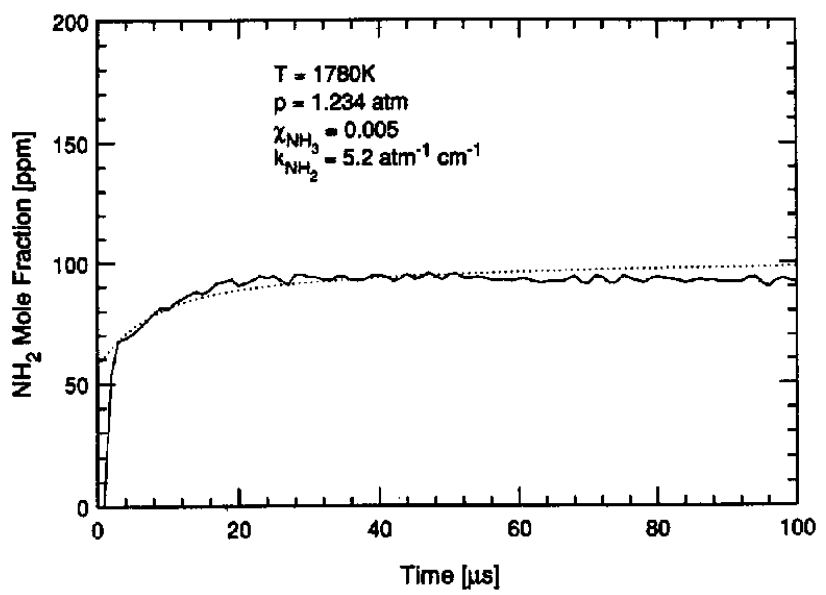

Fig. 4. $\mathrm{NH}_{3}$ photolysis experiments for the determination of the $\mathrm{NH}_{2}$ absorption coefficient showing $\mathrm{NH}_{2}$ mole fraction vs time. The experimental mole fraction is scaled by variation of the $\mathrm{NH}_{2}$ abeorption coefficient until agreement with the chemical kinetic model (dashed line) is achieved. The incremental mole fraction of $\mathrm{NH}_{2}$ (and $\mathrm{H}$ ) at $t=0$ is determined from the number of incident excimer laser photons. The reaction set is given in Table 1 .

3.1.2. $\mathrm{NH}_{3}$ pyrolysis experiments. In the second, kinetic calibration method, the $\mathrm{NH}_{2}$ absorption coefficient is obtained from pyrolysis experiments using mixtures of $0.1-0.9 \% \mathrm{NH}_{3}$ in $\mathrm{Ar}$. As before, the absorption coefficient is extracted from the measured $\mathrm{NH}_{2}$ absorption profile by matching it with the kinetic model of Table 1 . The absorption coefficient is varied until good agreement, particularly at early times, is found. Figure 5 shows a measurement performed at a reflected shock temperature of $2750 \mathrm{~K}$. In the pyrolysis experiments, $t=0$ is chosen to correspond to the arrival time of the reffected shock; in the data this appears as a spike on the $I$ trace due to the effects of beam steering as the shock front passes through the probe beam. Typically, $t=0$ is uncertain by $\pm 2-3 \mu \mathrm{sec}$, which is in all experiments $<10 \%$ of the time considered for the matching of experimental and simulated $\mathrm{NH}_{2}$ concentrations. In Fig. 5, the $\mathrm{NH}_{2}$ mole fraction is modelled well for about the first $50-60 \mu \mathrm{sec}$; beyond this time, the model predicts a slightly more rapid decay than is observed. Best agreement at early times is obtained with an absorption coefficient of $1.65 \mathrm{~cm}^{-1} \mathrm{~atm}^{-1}$.

The results from about 20 pyrolysis experiments in the range of $0.5-1$ atm and over the range $1950-3200 \mathrm{~K}$ are displayed in Fig. 6, together with the results from the 40 photolysis experiments. It is seen that the separate calibration strategies provide consistent results in the regime of overlapping temperature.

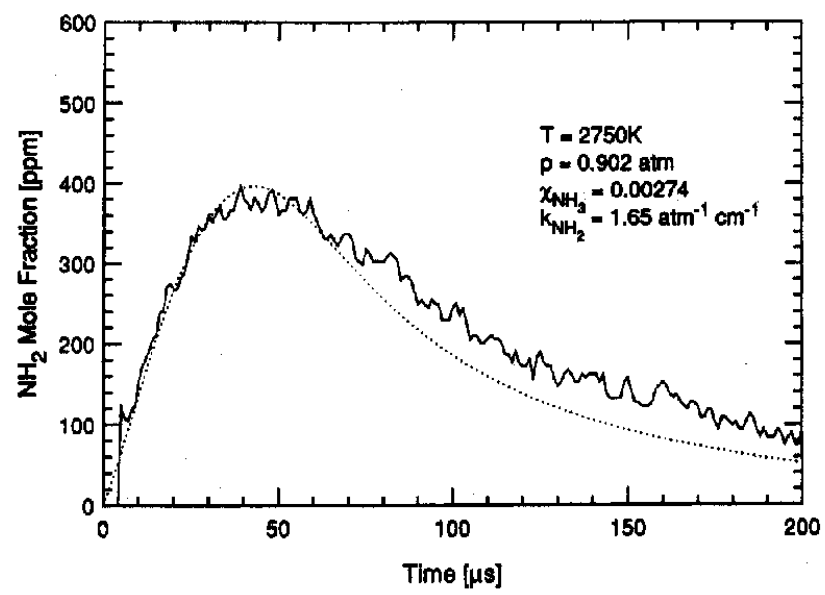

Fig. 5. $\mathrm{NH}_{3}$ pyrolysis experiments for the determination of $\mathrm{NH}_{2}$ absorption coefficient showing $\mathrm{NH}_{2}$ mole fraction vs time. The matching of experimental and simulated $\mathrm{NH}_{2}$ profiles is performed as in Fig. 4 by variation of the $\mathrm{NH}_{2}$ absorption coefficient. 


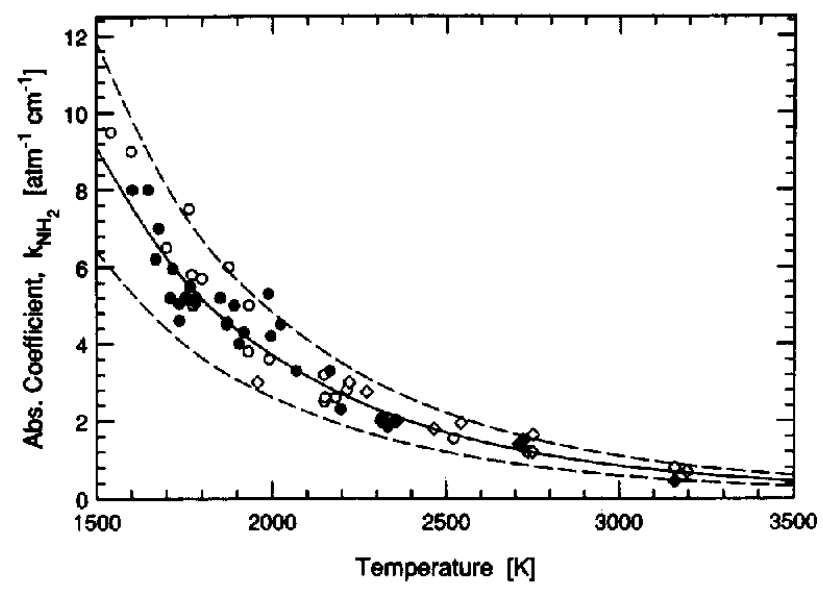

Fig. 6. $\mathrm{NH}_{2}$ line-center absorption coefficient vs temperature; $\mathrm{O}$-Collinear photolysis experiments; -end-wall photolysis experiments; $\diamond$-pyrolysis experiments at $P \sim 1$ atm; - pyrolysis experiments at $P \sim 0.5 \mathrm{~atm}$. The solid line is the calculated temperature dependence using a combined Voigt profile with a collision-broadening parameter $2 \gamma_{0}$ of $0.0175 \mathrm{~cm}^{-1} \mathrm{~atm}^{-1}$ at $2000 \mathrm{~K}$, scaled with $n=0.5$. The dashed lines correspond to the overall estimated error of $\pm 30 \%$ the determination of the absorption coefficient. The absorption coefficient can be calculated from the polynomial fit $k_{\mathrm{NH}_{2}}=3.322 \times 10^{10} / T^{3}+6.130 \times 10^{3} / T^{2}-1.302 \times 10^{3} / T$ with sufficient accuracy in the range $1600-3200 \mathrm{~K}$.

The scatter in Fig. 6 leads us to a discussion of the respective sources of error in the calibration experiments. The largest experimental error in the photolysis experiments arises from the measurement of the calibration factors which determine $I_{0,193}$. We estimate this error to be about $20 \%$. Within the limit, different series of experiments involving different calibration factors for collinear and end-wall photolysis give good agreement. Preparation of mixtures as well as the determination of reflected-shock temperature and pressure influence both photolysis and pyrolysis experiments in the same way; the resulting error is estimated to be $<10 \%$. Our $\mathrm{NH}_{3}$ absorption coefficient measured over all temperature is believed to be accurate to about $20 \%$.

Finally, the error contribution due to the kinetic modelling must be considered. Not all of the reactions listed in Table 1 are of equal importance for the determination of the $\mathbf{N H}_{2}$ absorption coefficient. Although a number of the reaction rate coefficients are only estimated, we use this fairly complete model for the analysis because it has been successfully tested in the simulation of experiments in $\mathrm{NH}_{3}$-containing flames, ${ }^{34}$ and has been verified in this laboratory in the prediction of $\mathrm{NH}$ profiles in $\mathrm{NH}_{3} / \mathrm{Ar}$ pyrolysis and photolysis in shock tube flows. Under all our experimental conditions, a study of contribution factors ${ }^{30}$ and a sensitivity analysis ${ }^{35}$ attributes the greatest influence on the $\mathrm{NH}_{2}$ profiles to the $\mathrm{NH}_{2}$ formation reactions (10) and (23), and to the $\mathrm{NH}_{2}$ consumption reactions (14) and (24). These four reactions are marked with double daggers in Table 1. Additionally, the reactions marked with one dagger play a role at specific conditions: the reactions (22) and (30) at long times in the photolysis experiments, and the reactions (5) and (17) at temperatures above $3000 \mathrm{~K}$ in the pyrolysis experiments.

From this discussion of the individual error sources, we can estimate overall uncertainty levels in typical experiments. In both collinear and end-wall photolysis, experimental errors on the order of $\pm 20 \%$ combine with uncertainties in the kinetic modelling to give an overall estimated error of $\pm 30 \%$, the collinear photolysis being slightly less accurate due to the inhomogeneous $\mathrm{NH}_{2}$ profiles at high $\mathrm{NH}_{3}$ concentrations. For the pyrolysis experiments, the overall uncertainty is determined to a greater extent by the uncertainties in the rate coefficients in the kinetic model. The most reliable experiments in this respect are the ones obtained at temperatures below $2500 \mathrm{~K}$, where at early times, only reaction (10), and subsequently reaction (23), are important. From a variational analysis of the reaction rate coefficients in this case, the error in the absorption coefficient is estimated to be $\pm 20 \%$. In the pyrolysis at 1 atm and $2500-3200 \mathrm{~K}$, the kinetic uncertainty is estimated to be $\pm 30 \%$ from a variational analysis of the reaction rate coefficients. Here, higher contributions from the $\mathrm{NH}_{2}$-consuming reactions with less well-known rate coefficients have some influence even at early times. 
In conclusion, the overall uncertainty of the $\mathrm{NH}_{2}$ absorption coefficient over the entire temperature range is estimated to be $\pm 30 \%$; this compares well with the scatter of individual experiments at a constant temperature in Fig. 6.

\subsection{Lineshape function and temperature dependence of the absorption coefficient}

The dependence of the absorption coefficient on temperature can be calculated from known modecular parameters. The absorption coefficient at the linecenter for a single line is given by (cgs units)

$$
k_{\mathrm{NH}_{2}}=\left(\pi e^{2} / m c^{2}\right)\left(N_{\mathrm{NH}_{2}} / p \chi_{\mathrm{NH}_{2}}\right) f_{\mathrm{B}} f \Phi(0, \mathbf{a}),
$$

where $e$ and $m$ are charge and mass of the electron, $c$ is the velocity of light, $N_{\mathrm{NH}_{2}}$ is the $\mathrm{NH}_{2}$ number density, $f$ is the oscillator strength for the monitored transition, and $f_{\mathrm{B}}$ is the Boltzmann fraction of the absorbing level. Here, $\Phi(x, a)$ is the lineshape function $(\mathrm{cm})$, normalized so that its integral over frequency $\left(\mathrm{cm}^{-1}\right)$ is one $\left(\int \Phi(x, \mathbf{a}) \mathrm{d} v=1\right) ; \Phi(0, \mathbf{a})$ is the value of this lineshape function at the linecenter and $x$ is the non-dimensional frequency

$$
x=2(\ln 2)^{1 / 2}\left(v-v_{0}\right) / \Delta v_{\mathrm{D}},
$$

where $v_{0}$ is the linecenter frequency, and $\Delta v_{\mathrm{D}}$ is the Doppler width. The Doppler width is obtained from

$$
\Delta v_{\mathrm{D}}=(8 R T \ln 2 / M)^{1 / 2}\left(v_{0} / c\right),
$$

where $R$ is the gas constant and $M$ is the molecular weight of $\mathrm{NH}_{2}$.

In Ref. 6, the calculation of the Boltzmann fraction is explained in detail for the same $\mathrm{NH}_{2}$ transition. We essentially follow their formalism and evaluate $f_{\mathrm{B}}$ from

$$
f_{\mathrm{B}}=(3 / 4)\left(2 J^{\prime \prime}+1\right) /\left(g^{\prime \prime} Q_{\mathrm{v}} Q_{\mathrm{r}}\right) \exp -\left(E_{T} / k T\right) \text {. }
$$

The factor (3/4) accounts for the nuclear statistics for the antisymmetric absorbing level, $g^{\prime \prime}$ is the electronic degeneracy of this level, $Q_{\gamma}$ and $Q_{\mathrm{r}}$ are the vibrational and rotational partition functions, respectively, and $E_{T}$ is the energy if the lower level. The partition functions are calculated in the usual way ${ }^{36}$ using moments of inertia and normal vibration frequencies of Refs. 6 and 20. Term values for the ground state are given by Dressler and Ramsay. ${ }^{20}$

3.2.1. Doublet splitting and intensity ratio. The lineshape function for the overlapped doublet $(090 \leftarrow 000) \Sigma^{p} Q_{1, N} 7$ transition is dependent on the splitting of the two components and their respective oscillator strengths and populations, as well as on the collision broadening. Green and Miller ${ }^{6}$ report a doublet splitting of $3.54 \mathrm{GHz}$, which they measured with a multipass optical arrangement in a low-pressure flame, where pure Doppler broadening was ensured. Under their conditions, the two spin components are barely resolved. They conclude from fitting the observed lineshape in their experiment that the observed ratio of the two peak intensities is due to the difference in population so that the oscillator strengths for transitions from both sublevels are equal.

Before we investigated the influence of collisional broadening, we determined the splitting and intensity ratio for the doublet under favorable conditions. For these experiments, the rapid scanning laser system was used with the shock tube as a static, room temperature cell. $\mathrm{NH}_{2}$ was produced by photolyzing pure $\mathrm{NH}_{3}$ in the end-wall configuration at a pressure of $<0.0025 \mathrm{~atm}$. Figure 7 shows a typical experimental trace. During the $\mathrm{NH}_{2}$ lifetime, the doublet structure is observed six times. The occurrence of the photolysis pulse is easily recognized by the sharp spike at $\sim 200 \mu \mathrm{sec}$ due to scattered excimer laser light. The order of the two doublet components reverses in time as the scanning is performed repeatedly forwards and backwards over the structure. The linewidth of the dye laser is negligibly small (nominal linewidth $<1 \mathrm{MHz}$ ) compared to the $\mathrm{NH}_{2}$ absorption line. The $2.00 \mathrm{GHz}$ etalon signal which is used to convert the time scale to a relative frequency scale is included as the dashed line. The noise on the background absorption signal is due to residual incomplete balancing of the frequency-modulated probe and reference beam intensities. The $\mathbf{N H}_{2}$ decay in Fig. 7 has to be accounted for in the data reduction, as the changing concentration influences the relative peak intensities. This is done by reconstructing the $\mathbf{N H}_{2}$ absorption profile from the measured peak heights, which is then used for the normalization. 


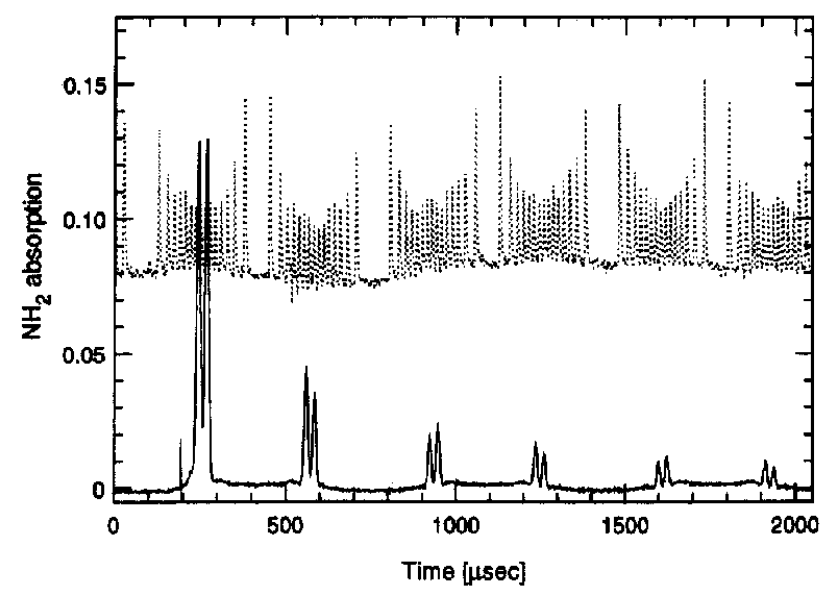

Fig. 7. $\mathrm{NH}_{2}$ scanning experiment performed at $295 \mathrm{~K}$ and $0.0023 \mathrm{~atm}$ in the end-wall photolysis configuration. The solid line shows six scanning cycles over the doublet lineshape after one photolysis pulse, with the direction of the scan reversing each time. The photolysis pulse occurs at $\sim 200 \mu$ sec and is indicated by the spike due to stray excimer light. The dashed line represents the $2.00 \mathrm{GHz}$ etalon trace, which is used to convert the time scale into frequency units.

The high-temperature absorption coefficient for the doublet is

$$
k_{\mathrm{NH}_{2}}=\left(\pi e^{2} / m c^{2}\right)\left(N_{\mathrm{NH}_{2}} / p \chi_{\mathrm{NH}_{2}}\right)\left[f_{\mathrm{B1}} f_{1} \Phi\left(x_{\max }, \mathbf{a}\right)+f_{\mathrm{B} 2} f_{2} \Phi\left(x_{\max }-\Delta x, \mathbf{a}\right)\right] \text {, }
$$

where the subscripts 1 and 2 identify the populations and the oscillator strengths for the two sublevels, $\Delta x$ corresponds to the doublet splitting, and $x_{\max }$ is the non-dimensional frequency that corresponds to the maximum of the combined doubled lineshape.

For the experiment at $295 \mathrm{~K}$ and $\sim 0.0025 \mathrm{~atm}$, we may assume the two lines to be entirely Doppler-broadened $(a=0)$. The splitting and the intensity ratio of the two peaks are then obtained from a best fit to the experimental lineshape, minimizing the residual least-squares area. From Green and Miller, ${ }^{6}$ the line with the higher intensity corresponds to $J_{1}^{\prime \prime}=N^{\prime \prime}+1 / 2$, the lower to $J_{2}^{\prime \prime}=N^{\prime \prime}-1 / 2$.

Figure 8 shows an example of such a lineshape analysis. The two components are resolved to a much higher extent then in the previous flame experiments. ${ }^{6}$ From about 20 of these
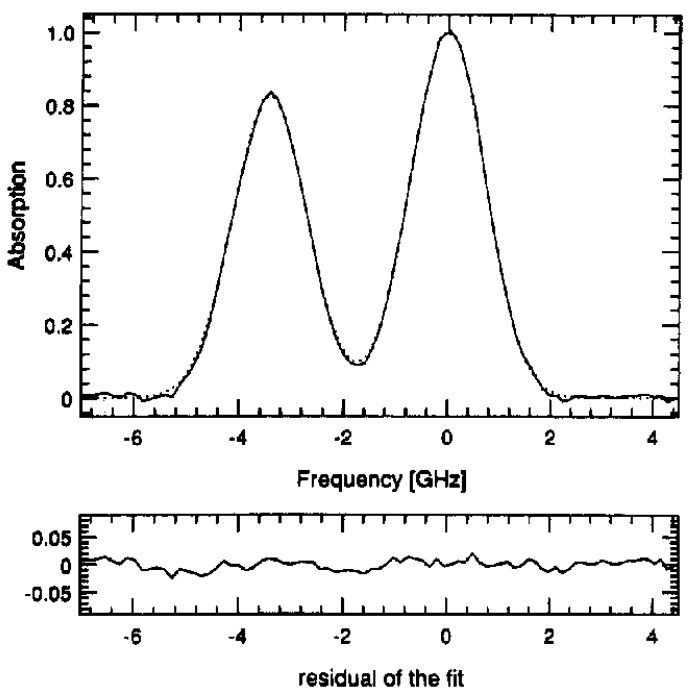

Fig. 8. Lineshape of the $\mathrm{NH}_{2}$ absorption doublet at $295 \mathrm{~K}$ and $0.0023 \mathrm{~atm}$ vs relative frequency. The absorption $\left(\ln I_{0} / I\right)$ is normalized to a maximum of 1 . The dotted line is the best fit to a combined Doppler function. In this case, the splitting of the two components is $3.42 \mathrm{GHz}$, the ratio of the oscillator strengths is 0.94 ; the higher peak corresponds to $J_{1}^{\prime \prime}=N^{\prime \prime}+1 / 2$. 
measurements, we determine the splitting to be $(3.44 \pm 0.02) \mathrm{GHz}$ and the ratio of the oscillator strengths to be $(0.93 \pm 0.03)$. The error limits are the deviations of the minimum and maximum values from the least-squares mean. These results are reasonably consistent with the ones of Green and Miller. ${ }^{6}$ With the better resolution of our experiment, the $3 \%$ smaller splitting we obtain is still within the error limits of their data. Our observation that the oscillator strengths for the two components are not exactly the same is expected by comparison with spin-split doublets for diatomic molecules such as $\mathrm{OH} .^{37}$

3.2.2. Collisional broadening. For the determination of the collision broadening parameters, scanning experiments were performed under reflected shock conditions at 1,2 , and $4 \mathrm{~atm}$ in either end-wall photolysis or pyrolysis. The temperature range was limited to $1800-2400 \mathrm{~K}$ because at higher temperature the $\mathrm{NH}_{2}$ concentration exhibited excessively steep gradients over the $\sim 300 \mu \mathrm{sec}$ necessary for one scanning cycle over the doublet. Because the wings of the line contain much of this lineshape information, an important consideration was that the absorption had to be maximized to achieve a good signal-to-noise ratio. Typically, 2-6\% peak absorption corresponding to $\leqslant 400 \mathrm{ppm} \mathrm{NH}$, was obtained, depending on the reflected shock pressure, with a signal-to-noise ratio of about $20-30$. This is significantly less than encountered for similar $\mathrm{OH}$ experiments ${ }^{19}$ where $30-50 \%$ absorption, corresponding to $\leqslant 100 \mathrm{ppm} \mathrm{OH}$, were obtained. Figure 9(a) shows the lineshape of the overlapping doublet at $1970 \mathrm{~K}$ and $1.78 \mathrm{~atm}$. In contrast to the low temperature and pressure case of Fig. 8, the doublet structure is barely recognizable; it is manifest only in the asymmetric nature of the profile at the peak.

Using the splitting and the intensity ratio as determined before, the experimental lineshape is fitted to a combined Voigt function for the two doublet components. The usual collision-broadened lineshape function is given by a (suitably normalized) Voigt profile, ${ }^{38}$ with the ratio of collisional and Doppler broadening expressed as the a parameter,

$$
\mathrm{a}=\left(\Delta v_{\mathrm{C}} / \Delta v_{\mathrm{D}}\right)(\ln 2)^{1 / 2} \text {. }
$$

The collision broadened halfwidth (FWHM) $\Delta v_{\mathrm{C}}$ is given by

$$
\Delta v_{\mathrm{C}}=C N_{\mathrm{tot}} \bar{\sigma} v \text {, }
$$

where the effective cross section for broadening collisions $\sigma$ (a weighted average for the different collider species) may be a function of temperature, $C$ is a constant independent of temperature,
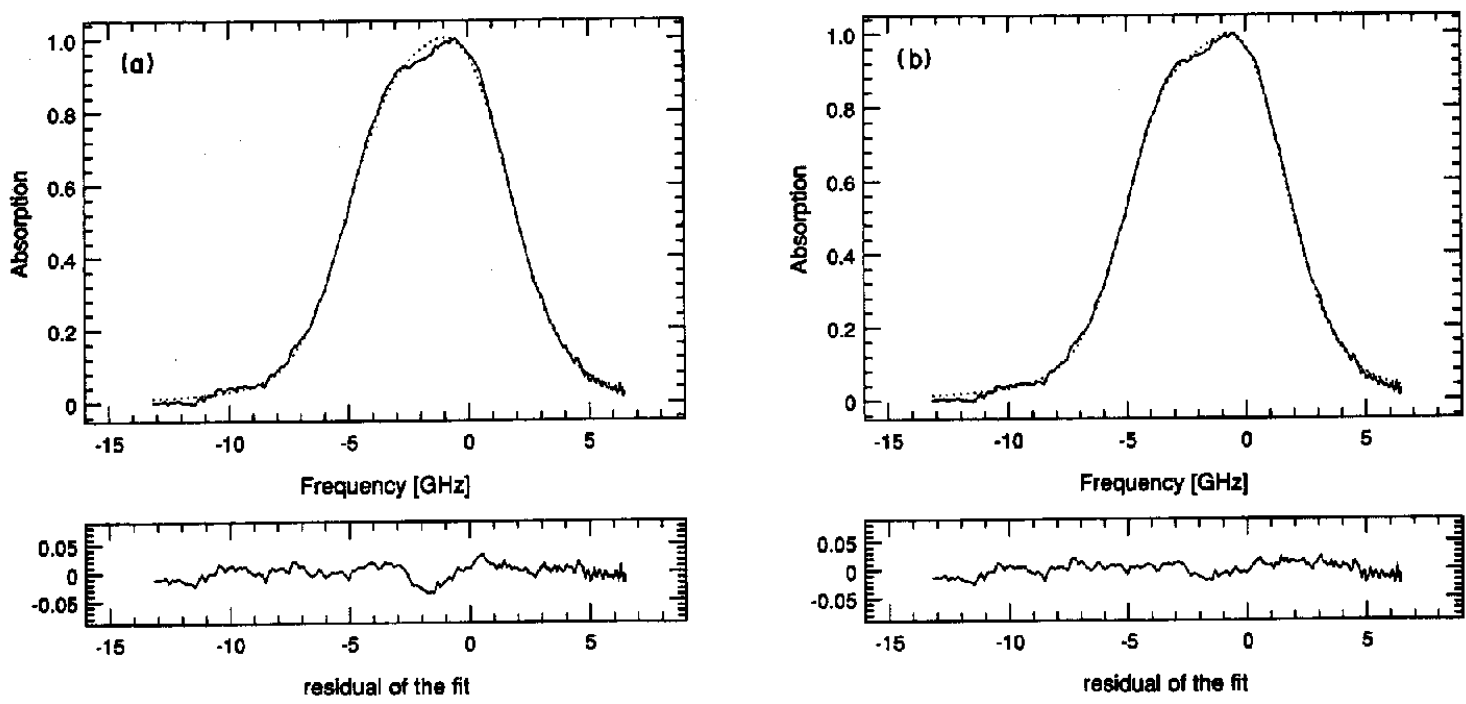

Fig. 9. Lineshape of the $\mathrm{NH}_{2}$ absorption doublet at $1970 \mathrm{~K}$ and $1.78 \mathrm{~atm}$ vs relative frequency. The absorption $\left(\ln I_{0} / I\right)$ is normalized to a maximum of 1 . (a) The dotted line is the best fit to a combined Voigt function with $a=0.248$. The residual of the fit exhibits a slight systematic deviation at the center of the doublet. (b) The dotted line is the best fit to a combined Galatry function with $a=0.272$ and $z=0.16(r=1.7)$. The residual shows an improved fit at the center of the doublet. 
and $v$ is the average velocity which scales with $T^{1 / 2}$. The commonly employed collisional broadening parameter $2 \gamma$ is related to $\Delta v_{\mathrm{C}}$ by

$$
\Delta v_{\mathrm{C}}=\Sigma_{i} 2 \gamma_{i} p_{i},
$$

where $p_{i}$ is the partial pressure (atm) of the broadening species $i$. In our experiments with $\mathrm{NH}_{3}$ mole fractions below $1 \%$, we assume the broadening to be entirely governed by the background gas Ar. For the assumption of a hard-spheres collision model, $2 \gamma$ scales with $T^{-1 / 2}$; if $\sigma$ decreases with increasing temperature, as is commonly observed, the exponent in $T$ is smaller than $-1 / 2$.

In the fitting procedure, the best fit is determined by the least-squares residual area, with $a=a_{1}=a_{2}$ being the only free parameter. For the example of Fig. 9(a), the calculated lineshape is shown as the dotted line. In this case, the a parameter is 0.248 , leading to a broadening parameter $2 \gamma$ of $0.0223 \mathrm{~atm}^{-1} \mathrm{~cm}^{-1}$. The residual of the fit is about $\pm 2 \%$, twice as much as obtained for the experiment in Fig. 8 where no shock was performed.

The residual in Fig. 9(a) exhibits a systematic structure which was found to be similar in all of the experiments. In previous $\mathrm{OH}$ experiments in this laboratory, ${ }^{19,39,40}$ systematic deviations from Voigt lineshapes have been found in connection with collisional narrowing effects. ${ }^{41}$ More refined lineshape models that the commonly adopted Voigt profile can account for the interdependence of the thermal motion of the molecules (Doppler broadening) and the collisional perturbation of their energy distribution by, for example, considering velocity changes by collisions or velocitydependent collisional cross sections. Detailed descriptions of such lineshape models can be found in Refs. 40, 42, and 43.

Figure 9(b) shows the same experiment as Fig. 9(a); this time, a combined Galatry ${ }^{44}$ profile is used in the analysis. The best-fit is obtained with an a parameter of 0.272 (corresponding to a value of $2 \gamma$ of $0.0245 \mathrm{~cm}^{-1} \mathrm{~atm}^{-1}, 10 \%$ higher than before) and a collisional narrowing parameter $z$ of 0.16 , when $a$ and $z$ are iteratively changed until the least-squares area is minimized. The residual in Fig. 9(b) shows an improved fit at the center of the doublet, and the residual least-squares area is $30 \%$ lower than before. Similarly better quality of the fits was obtained for all experiments which were analyzed with a combined Galatry profile.

In the soft-collision Galatry lineshape model, the parameter $z$ describes the restriction (narrowing) of the Doppler velocity distribution as seen by the absorbing beam; $z$ is essentially the ratio of the frequency of velocity-changing collisions $\beta$ to the Doppler width $\Delta v_{D} . \beta$ is usually ${ }^{42,43}$ related to an optical diffusion coefficient, which is of the same order of magnitude as the mass diffusion coefficient obtained from transport properties. Significant influence of motional narrowing has been observed mainly for molecules which exhibit large rotational level spacings, such as $\mathrm{H}_{2}$, or the diatomic hydrides such as the $\mathrm{OH}$ radical. ${ }^{40,42,43}$ This fact suggests the likelihood of measurable contributions to collisional narrowing for $\mathrm{NH}_{2}$ lineshapes. With the mass diffusion coefficient quoted in Ref. 45 for diffusion of $\mathrm{ND}_{2}$ in $\mathrm{Ar}$, scaled to our temperatures and pressures, we obtain a crude estimate of the narrowing parameter $z$ of 0.35 for the experiment in Fig. 9. This is only about a factor of 2 different from the value determined from a best-fit to the experimentally observed lineshape. The ratio $r=\mathbf{a} / z$ of the broadening and narrowing parameters is independent of pressure and only a weak function of temperature. From seven experiments at $0.95-3.5 \mathrm{~atm}$ and $1950-2300 \mathrm{~K}$, we find $r=1.8 \pm 0.5$; this is almost exactly the same value as reported for a Galatry analysis of $\mathrm{OH}$ lineshapes broadened by $\mathrm{Ar}^{40}$ in the same temperature range. This surprising similarity might be due to the common nature of both radicals as hydrides with large rotational constants.

Although these observations seem to suggest that an interpretation of the doublet $\mathrm{NH}_{2}$ lineshape which includes motional narrowing would be more appropriate, the comparatively low signal-tonoise level of the data precludes a more thorough analysis. For the purpose of our experiments, we consider the description by a combined Voigt lineshape, leading to values of $2 \gamma$ which are on the average about $10 \%$ lower than inferred with the Galatry analysis, as sufficiently accurate.

Figure 10 shows the collision broadening parameter $2 \gamma$ as obtained from the fitting of the experimental lineshapes using a combined Voigt profile in Eq. (7). The open symbols refer to a series of experiments at 1,2, and 4 atm; an additional later set of experiments performed at $2 \mathrm{~atm}$ is represented by the solid triangles. A slight systematic difference of $\sim 25 \%$ is observed which reflects the precision of this experiment. The absolute values of $2 \gamma$ in Fig. 10 can be compared to 


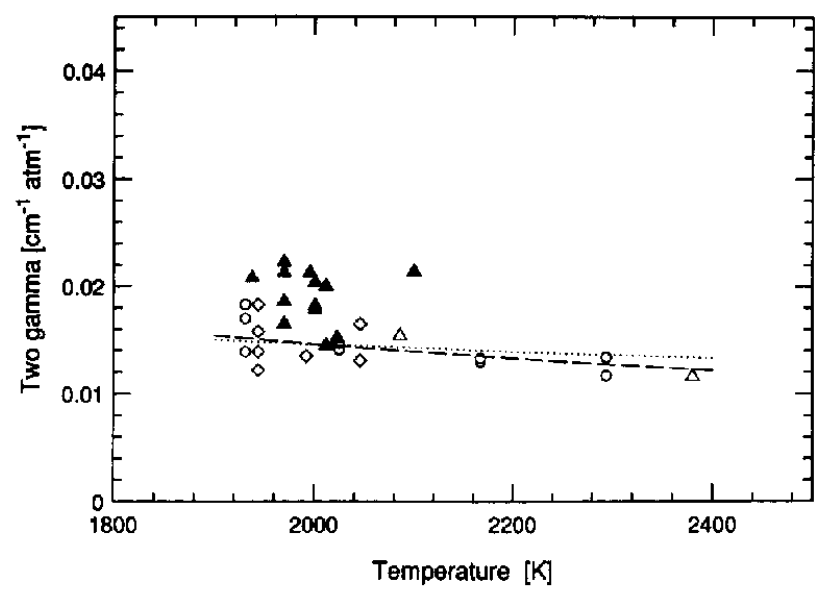

Fig. 10. Collision broadening parameter for the broadening of $\mathrm{NH}_{2}$ by $\mathrm{Ar}$ vs temperature. Solid and open symbols correspond to two different series of experiments. Circles $-P \sim 1 \mathrm{~atm}$; triangles $-P \sim 2 \mathrm{~atm}$; diamonds $-P \sim 4 \mathrm{~atm}$. The lines are calculated with $n=0.5$ (dashed) and $2 \gamma_{0}=0.0146 \mathrm{~cm}^{-1} \mathrm{~atm}^{-1}$ at $2000 \mathrm{~K}$.

the broadening of $\mathrm{OH}^{19}$ and $\mathrm{NH}^{46}$ by Ar where $2 \gamma$ determined for these diatomics is about a factor of 2 higher than for $\mathrm{NH}_{2}$. We presently have no firm explanation for this difference.

The extrapolation of $2 \gamma$ to different temperatures is usually performed using the relation

$$
2 \gamma=2 \gamma_{0}\left(T_{0} / T\right)^{n},
$$

with $2 \gamma_{0}$ being the value at the reference temperature $T_{0}$, and $n$ typically between 0.5 and 1 . In view of the relatively small temperture range and the significant scatter, we do not attempt to determine $n$ from the experimental data. The two lines in Fig. 10 are calculated with $n=0.5$ (dotted) and $n=1.0$ (dashed) and $2 \gamma_{0}=0.0146 \mathrm{~cm}^{-1} \mathrm{~atm}^{-1}$ at $2000 \mathrm{~K}$. Both lines seem to give equally adequate representations of the earlier data set (open symbols). It shall be mentioned, however, that the value of $n$ would likely decrease if a Galatry lineshape were used, ${ }^{40}$ as the restrictions on the thermal motion would be more pronounced at higher temperatures where the Doppler contribution to the lineshape is larger. For the extrapolation of $2 \gamma$ in the temperature range of $1600-3200 \mathrm{~K}$, we suggest an average value of $2 \gamma_{0}=0.0175 \mathrm{~cm}^{-1} \mathrm{~atm}^{-1}$ at $2000 \mathrm{~K}$ (obtained from both sets of experiments) and $n=0.5$. The magnitude of errors caused by this assumption in the temperature dependence of the absorption coefficient is discussed in the next section.

3.2.3. Temperature dependence of the absorption coefficient. The temperature dependence of the maximum absorption coefficient for the doublet is calculated using Eqs. (6) and (7) and the known lineshape function. The Voigt a parameter is obtained by extrapolation of $2 \gamma_{0}$ with Eq. (11) with $n=0.5$. Apart from the number density $N_{\mathrm{NH}_{2}}=N_{\text {tot }} \chi_{\mathrm{NH}_{2}}$, only the Boltzmann fraction and the combined Voigt function are dependent on temperature in $\mathrm{Eq}$. (7). The sensitivity of the temperature dependence of the absorption coefficient to different lineshape assumptions is shown in Fig. 11. The maximum of the doublet assuming a Voigt function for the lineshapes is displayed for two cases: scaling $2 \gamma$ with $n=0.5$ (solid line) or with $n=1$ (dashed line). For comparison, the maximum of the doublet for Doppler lineshapes is included.

For the calculations the maximum of the lineshape function is always used. At high temperatures, the maximum occurs approximately at the centerline frequency of the doublet; this frequency was determined empirically and was used throughout the experiments. At low temperatures the two peaks are no longer blurred, and a small error is introduced by keeping the laser fixed on this centerline frequency because the maximum is shifted toward the $J_{1}^{\prime \prime}$ peak. At $1500 \mathrm{~K}$ the difference between the maximum absorption coefficient and the absorption coefficient at the centerline frequency is $\sim 6 \%$; at $1700 \mathrm{~K}$, it is already $<3 \%$. Thus these errors are not significant in the temperature range above $1600 \mathrm{~K}$. Of course, this assumption would not be adequate at temperatures approaching room temperature where the doublet is fully resolved (see Fig. 8).

As shown in Fig. 11, the temperature dependence of the absorption coefficient is not very sensitive to the particular lineshape model. A difference of only about $2 \%$ is obtained using the 


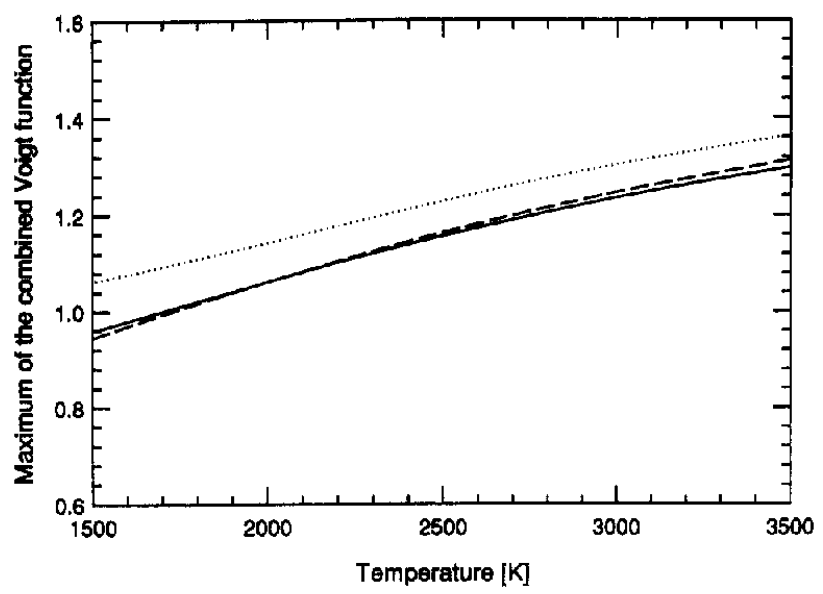

Fig. 11. Maximum of the combined Voigt function vs temperature. The solid line refers to $2 \gamma_{0}=0.0175 \mathrm{~cm}^{-1} \mathrm{~atm}^{-1}$ at $2000 \mathrm{~K}, n=0.5$; the dashed line refers to the same $2 \gamma_{0}$ value with $n=1.0$; the dotted line represent the pure Doppler limit.

measured $2 \gamma_{0}$ at $2000 \mathrm{~K}$ with two different values of $n, 0.5$ and 1.0 . With $2 \gamma_{0}$ increased by $25 \%$ (corresponding to the experimental error), the maxima decrease by $<2 \%$. Neglecting the collision broadening entirely changes the maxima by $<10 \%$. A comparison with a combined Galatry lineshape is not included in Fig. 11, since precise information on the temperature dependence of the $z$ parameter is lacking. Typically the maxima for Galatry profiles with a and $z$ on the order of our experimental values increase by $\sim 5 \%$ in comparison with the maxima of the respective Voigt functions. ${ }^{42,43}$

The calculated temperature dependence using the maxima given by the solid line in Fig. 11 agrees well with the experimented data shown in Fig. 6. The potential errors associated with our incomplete information on the temperature dependence of the collision broadening are much less than the experimental uncertainties discussed in Sec. 3.1.2, as the lineshape is strongly inftuenced by the Doppler contribution under our conditions. The use of the absorption coefficient in Fig. 6 at lower or moderately higher pressures does not therefore introduce large systematic errors.

As the temperature dependence of the absorption coefficient is sufficiently complex to preclude an easy calculation of the absorption coefficient at a particular temperature, we recommend a simple polynomial fit to the experimental data, $k_{\mathrm{NH}_{2}}=3.322 \times 10^{10} / T^{3}+6.130 \times 10^{5} / T^{2}-1.302 \times$ $10^{3} / T$, in the temperature range of $1600-3200 \mathrm{~K}$. The absorption coefficient can be calculated for different pressures using the lineshape function with the measured collision broadening parameters. Below 1 atm this might not be necessary in view of the small difference between the combined Voigt and combined Doppler function, but pressure effects should be taken into account above 1 atm.

\subsection{Oscillator strength}

The oscillator strength for the transition can be evaluated in two ways from our experimental results. First, it can be obtained from Eq. (7) using the combined Voigt lineshape inferred from the scanning experiments. Secondly, the measured lineshapes can be integrated directly. The result of this latter method is independent of the collision broadening parameter, but the number of useful experiments is limited, as discussed in Sec. 3.2.2.

Rearrangement of Eq. (7) allows one to solve for $f_{1}$, using 0.93 as the ratio $f_{1} / f_{2}$ of oscillator strengths as determined from the room temperature scanning experiments. The evaluation of all 59 calibration experiments gives $f_{1}=(6.59 \pm 0.99) \times 10^{-5}$; the error limit is $1 \mathrm{SD}$. The 41 photolysis experiments lead to $f_{1}=(6.62 \pm 0.93) \times 10^{-5}$, and the 18 pyrolysis experiments to $f_{1}=(6.53 \pm 1.13) \times 10^{-5}$. The average of $f_{1}$ from 17 collinear and 24 end-wall photolysis experiments, as well as from 10 pyrolysis experiments near $1 \mathrm{~atm}$ and eight pyrolysis experiments near $0.5 \mathrm{~atm}$, deviate by $0.8,2.5,5.2$, and $8.6 \%$, respectively, from the average $f_{1}$ determined from all 59 experiments. The differences due to systematic errors of either $\mathrm{NH}_{2}$ generation scheme are much less than the experimental scatter of $\pm 30 \%$. To facilitate the comparison with previous experi- 
ments, the average oscillator strength $f$ for the entire doublet transition is calculated from

$$
f=\left(f_{1} f_{\mathrm{B} 1}+f_{2} f_{\mathrm{B} 2}\right) /\left(f_{\mathrm{B} 1}+f_{\mathrm{B} 2}\right),
$$

which yields $f=(6.36 \pm 0.96) \times 10^{-5}$.

The lineshapes from eight experiments at 1 atm and $1900-2300 \mathrm{~K}$ can be integrated to obtain the area of the doublet. Only the 1 atm data can be treated in this fashion because the line-center absorption coefficient necessary to place the measured lineshape on an absolute scale is only measured at $1 \mathrm{~atm}$. The oscillator strength for the doublet is then calculated from

$$
f=\left(m c^{2} / \pi e^{2}\right)\left[p \chi_{\mathrm{NH}_{2}} / N_{\mathrm{NH}_{2}}\left(f_{\mathrm{B} 1}+f_{\mathrm{Bz}}\right)\right] k_{\mathrm{NH}_{2}} \int G_{\gamma}(x) \mathrm{d} x,
$$

where $G_{v}(x)$ is the experimental combined lineshape normalized so that the maximum, $G_{v}\left(x_{\max }\right)$, is one (cf. Fig. 9); multiplication by $k_{\mathrm{NH}_{2}}$ scales this to the correct centerline value. We obtain $f=(6.13 \pm 0.14) \times 10^{-5}$ from the evaluation of these experiments. The high statistical precision in this case should not be overinterpreted as we use the average value of $k_{\mathrm{NH}_{2}}$ (as given by the solid line in Fig. 6) in the evaluation. The oscillator strength calculated from the integration of measured lineshapes is only $4 \%$ lower than the value determined above; this insignificant deviation can be due to residual truncation errors in the integration.

We recommend an oscillator strength of $f=(6.35 \pm 1.90) \times 10^{-5}$ from consideration of all experiments including the results of the lineshape integration. The error limits of $\pm 30 \%$ correspond to the overall experimental uncertainty in the absorption coefficient.

This oscillator strength is in excellent agreement with the one of Dean et al $1^{8}\left(f=6.4 \times 10^{-5}\right)$ obtained from flame experiments. However our determination is essentially independent of thermodynamic data such as the heats of formation of $\mathrm{NH}$ or $\mathrm{NH}_{2}$, whereas the result from the flame experiments is very sensitive to these quantities. A recent investigation ${ }^{17}$ gives a recommendation for these heats of formation from a critical review of the literature. Re-evaluated with these values, the oscillator strength of Ref. 8 would decrease to $(3.9 \pm 1.2) \times 10^{-5}$ or $(5.3 \pm 1.6) \times 10^{-5}$, depending on the particular experiments considered. ${ }^{17}$ The higher value would still be consistent with our error limits. Our oscillator strength does not agree with the most recent value $e^{9,17}$ of $(1.96 \pm 0.20) \times 10^{-4}$ obtained on a theoretical basis. + In his calculations, Anderson ${ }^{17}$ uses the electronic transition moment of Mayama et $\mathrm{al}^{47}$ who evaluate their own lifetime measurements together with those of two previous studies. ${ }^{23,48}$ The resulting electronic transition moment ${ }^{47}$ is in very good agreement with the one calculated by Peyerimhoff and Buenker. ${ }^{49}$ It is not intended in this paper to review details of the calculations of Ref. 17. We presently do not understand, however, why that careful study leads to a result which differs so much from our experimentally determined oscillator strength.

\section{SUMMARY}

We have developed a cw laser-absorption technique for the quantitative detection of $\mathrm{NH}_{2}$ under shock tube conditions. The center-line absorption coefficient for the $\mathbf{N H}_{2}$ $\tilde{A}^{2} A_{1} \leftarrow \hat{X}^{2} B_{1}(090 \leftarrow 000) \Sigma^{p} Q_{1, N} 7$ transition was obtained from different calibration methods where known amounts of $\mathrm{NH}_{2}$ were generated either by $\mathrm{NH}_{3}$ pyrolysis or by photolysis of $\mathrm{NH}_{3}$ with an ArF excimer laser at $193 \mathrm{~nm}$. The pyrolysis experiments covered the temperature range $1950-3200 \mathrm{~K}$. The photolysis experiments were performed using two different geometries of photolysis and probe lasers at temperatures of $1600-2500 \mathrm{~K}$. All experiments agree well over the entire temperature range. From chemical kinetic uncertainties in the analysis of $\mathbf{N H}_{2}$ absorption traces, and uncertainties in the determination of the incident number of photolysis laser photons, the overall error in the absorption coefficient is estimated to be $\pm 30 \%$.

The monitored transition is an overlapping spin-split doublet. We used rapid scanning of the absorption laser ${ }^{19}$ to obtain information on the lineshape of the doublet. From excimer laser photolysis experiments at room temperature and low pressure, we determined the doublet splitting

†n a preliminary study,,$^{50}$ we reported an estimate of the oscillator strength in closer agreement with that of Anderson et al. ${ }^{9,17}$ However, in the early analysis of the photolysis data, the rapid production of $\mathrm{NH}_{2}$ by reaction 23 was neglected; furthermore, in the absence of lineshape information, the collision broadening was overestimated. Both effects lead to a change of the oscillator strength in the same direction. 
and the ratio of the oscillator strengths with higher resolution than previously reported from low-pressure flame experiments. ${ }^{6}$ Scanning experiments in shock-heated $\mathrm{NH}_{3} / \mathrm{Ar}$ mixtures at pressures of 1-4 atm provided the collision broadening parameter in the temperature range of $1900-2400 \mathrm{~K}$. The broadening is about a factor of 2 lower than values for $\mathrm{OH}$ broadening by Ar. ${ }^{19}$ The reason for this difference is unclear. For the determination of the collision broadening parameter, the experimental lineshapes were fitted with a combined Voigt function for the doublet. Slightly better fits were obtained when combined Galatry profiles were used instead. The collisional narrowing parameter determined from the best fit is of the same order of magnitude as the estimate based on the mass diffusion coefficient of $\mathrm{ND}_{2}$ in Ar of Ref. 45. The ratio of collision broadening to narrowing, which is expected to be independent of pressure and only weakly dependent on temperature, was found to be almost constant for the pressure and temperature range investigated and in good agreement with the value reported for $\mathrm{OH} .{ }^{40}$ Although a Galatry profile seemed to be more appropriate in the lineshape analysis, the use of the combined Voigt function was considered as sufficiently accurate for the purposes of our experiments.

With the information on the splitting oscillator strength ratio, and collision broadening obtained this way, we calculated the temperature dependence of the absorption coefficient and found this to be in very good agreement with the experimental data. As the lineshape is mainly determined by the thermal velocity distribution rather than by collisional broadening at our pressures, assumptions concerning the scaling law for the extrapolation of the collision broadening parameter to the entire temperature range were of negligible influence on the temperature dependence of the absorption coefficient.

From the lineshapes measured at $1 \mathrm{~atm}$ and temperatures of $1900-2300 \mathrm{~K}$ in combination with the line-center absorption coefficient, we determined the average oscillator strength for the doublet. Similarly, the oscillator strength was evaluated from all calibration experiments in the temperature range of $1600-3200 \mathrm{~K}$ using the combined Voigt function for the doublet. The averages of both series agree within $4 \%$. The difference between the average of the oscillator strength obtained from the photolysis experiments in comparison with that determined from the pyrolysis experiments is $<2 \%$. This demonstrates that systematic errors affecting either method of calibration have only insignificant influence on the results. From all experiments, we determined an average oscillator strength for the doublet of $(6.35 \pm 1.90) \times 10^{-5}$; the error limits of $\pm 30 \%$ are due to the overall uncertainty in the absorption coefficient. The oscillator strength is in excellent agreement with a value determined from flame experiments. ${ }^{8}$ However, in the previous determination the oscillator strength depended in a sensitive way on the heats of formation of $\mathrm{NH}$ or $\mathrm{NH}_{2}$, whereas our evaluation is essentially independent of such thermodynamic quantities. Our oscillator strength does not agree with the most recent theoretically calculated value, ${ }^{17}$ which is about a factor of 3 higher. We have no explanation for this discrepancy.

The absorption coefficient for $\mathrm{NH}_{2}$ is 1-3 orders of magnitude lower than those for diatomic radicals of combustion relevance, such as $\mathrm{OH}, \mathrm{NH}, \mathrm{CH}$, or $\mathrm{CN}$. This result implies a significantly lower detection sensitivity, particularly in comparison with diatomic radicals which allow narrow-band laser-absorption monitoring on a bandhead, e.g., NH or CN. Whereas sub-ppm detection limits are in principle attainable for those radicals in similar shock-tube experiments, the detection limit for $\mathrm{NH}_{2}$ as a triatomic molecule is on the order of $10-50 \mathrm{ppm}$, depending on the specific conditions. In spite of this reduced sensitivity, this direct quantitative diagnostic of $\mathrm{NH}_{2}$ is likely to be of considerable value in kinetic investigations of combustion-relevant systems. Such studies are presently being performed in this laboratory.

Acknowledgements - This work was supported by the Department of Energy, Office of Basic Energy Sciences, Division of Chemical Sciences. One of us (KKH) achnowledges support for her stay in the US from the German Aerospace Research Establishment (DFVLR). Furthermore, she wishes to thank J. A. Miller, Sandia National Laboratory, Livermore, CA, for discussions about the kinetic modelling and W. R. Anderson, Ballistic Research Laboratory, Aberdeen, MD, for a stimulating exchange of data and opinions concerning $\mathrm{NH}_{2}$ spectroscopy.

\section{REFERENCES}

1. J. A. Miller, M. D. Smooke, R. M. Green, and R. J. Kee, Combust. Sci. Technol. 34, 149 (1983).

2. J. A. Miller, M. C. Branch, and R. J. Kee, Combust. Flame 43, 81 (1981).

3. S. Saliman and R. K. Hanson, Combust. Sci. Technol. 23, 225 (1980).

4. M. A. Kimball-Linne and R. K. Hanson, Combust. Flame 64, 337 (1986). 
5. R. A. Copeland, D. R. Crosley, and G. P. Smith, Twentieth Symposium (Int.) on Combustion, p. 1195, The Combustion Institute, Pittsburgh, PA (1984).

6. R. M. Green and J. A. Miller, JQSRT 4, 313 (1981).

7. M. S. Chou, A. M. Dean, and D. Stern, J. Chem. Phys 76, 5334 (1982.)

8. A. M. Dean, M. S. Chou, and D. Stern, Int. J. Chem. Kinet. 16, 633 (1984).

9. K. N. Wong, W. R. Anderson, J. A. Vanderhoff, and A. J. Kotlar, J. Chem. Phys. 86, 93 (1987).

10. W. Hack, H. Schacke, M. Schröter, and H. Gg. Wagner, Seventeenth Symposium (Int.) on Combustion, p. 505, The Combustion Institute, Pittsburgh, PA (1979).

11. P. Dransfeld, W. Hack, H. Kurzke, F. Temps, and H. Gg. Wagner, Twentieth Symposium (Int.) on Combustion, p. 655, The Combustion Institute, Pittsburgh, PA (1984).

12. R. Patrick and D. M. Golden, J. Phys. Chem. 88, 491 (1984).

13. R. Lesclaux, in Reviews of Chemical Intermediates, Vol. 5, p. 347, Elsevier, Amsterdam (1984).

14. T. R. Roose, R. K. Hanson, and C. H. Kruger, Eighteenth Symposium (Int.) on Combustion, p. 853, The Combustion Institute, Pittsburgh, PA (1981).

15. S. Salimian, R. K. Hanson, and C. H. Kruger, Combust. Flame 56, 83 (1984).

16. E. C. Rea, Jr., S. Salimian, and R. K. Hanson, Appl. Opt. 23, 1691 (1984).

17. W. R. Anderson, J. Phys. Chem., in press (1989).

18. D. F. Davidson, A. Y. Chang, and R. K. Hanson, Twenty-Second Symposium (Int.) on Combustion, The Combustion Institute, Pittsburgh, PA, in press (1989).

19. E. C. Rea, Jr., A. Y. Chang, and R. K. Hanson, JQSRT 37, 117 (1987).

20. K. Dressler and D. A. Ramsay, Phil. Trans. R. Soc. Lond. A251, 553 (1959).

21. H. Okabe, Photochemistry of Small Molecules, Wiley, New York, NY (1978).

22. V. M. Donnelly, A. P. Baronavski, and J. R. McDonald, Chem. Phys. 43, 271 (1979).

23. V. M. Donnelly, A. P. Baronavski, and J. R. McDonald, Chem. Phys. 43, 283 (1979).

24. R. D. Kenner, F. Rohrer, and F. Stuhl, J. Chem. Phys. 86, 2036 (1987).

25. R. D. Kenner, F. Rohrer, R. K. Browarzik, A. Kaes, and F. Stuhl, Chem. Phys. 118, 141 (1987).

26. R. D. Kenner, R. K. Browarzik, and F. Stuhl, Chem. Phys. 121, 457 (1988).

27. D. F. Davidson, A. Y. Chang, K. Kohse-Höinghaus, and R. K. Hanson, JQSRT, in press (1989).

28. K. Watanabe, J. Chem. Phys. 22, 1564 (1954).

29. M. Suto and L. C. Lee, J. Chem. Phys. 78, 415 (1983).

30. R. J. Kee, J. A. Miller, and T. H. Jefferson, "CHEMKIN: A General Purpose Problem Independent, Transportable FORTRAN Chemical Kinetics Code Package," Sandia National Laboratory Report SAND80-8003, Livermore, CA (1980).

31. P. Marshall and A. Fontijn, J. Chem. Phys. 85, 2637 (1986).

32. J. V. Michael, J. W. Sutherland, and R. B. Klemm, J. Phys. Chem. 90, 497 (1986).

33. W. Hack, P. Rouveirolles, and H. Gg. Wagner, J. Phys. Chem. 90, 2505 (1986).

34. J. A. Miller, Sandia National Laboratory, Livermore, private communication.

35. A. E. Lutz, R. J. Kee, and J. A. Miller, "SENKIN: a FORTRAN Program for Predicting Homogeneous Gas Phase Chemical Kinetics with Sensitivity Analysis," Sandia National Laboratory Report SAND87-8248, Livermore, CA (1988).

36. G. Herzberg, Infrared and Raman Spectra of Polyatomic Molecules, Van Nostrand-Reinhold, New York, NY (1945).

37. A. Goldman and J. R. Gillis, JQSRT 25, 111 (1981).

38. A. C. G. Mitchell and M. W. Zemansky, Resonance Radiation and Excited Atoms, Cambridge University Press (1961).

39. E. C. Rea, Jr., A. Y. Chang, and R. K. Hanson, JQSRT 41, 29 (1989).

40. E. C. Rea, Jr., A. Y. Chang, and R. K. Hanson, "Motional Narrowing Effects in Spectral Line Profiles of $\mathrm{OH}, "$ in preparation (1989).

41. R. H. Dicke, Phys. Rev. 89, 472 (1953).

42. P. L. Varghese, Ph.D. Thesis, Stanford University, CA (1983).

43. P. L. Varghese and R. K. Hanson, Appl. Opt. 23, 2376 (1984).

44. L. Galatry, Phys. Rev. 122, 1218 (1961).

45. A. I. Chichinin and L. N. Krasnoperov, Chem. Phys. Lett. 115, 343 (1985).

46. A. Y. Chang and R. K. Hanson, JQSRT, in press (1989).

47. S. Mayama, S. Hiraoka, and K. Obi, J. Chem. Phys. 80, 7 (1984).

48. J. B. Halpern, G. Hancock, M. Lenzi, and K. H. Welge, J. Chem. Phys. 63, 4808 (1975).

49. S. D. Peyerimhoff and R. J. Buenker, Can. J. Chem. 57, 3182 (1979).

50. K. Kohse-Höinghaus, D. F. Davidson, and R. K. Hanson, Western States Section of the Combustion Institute, Spring Meeting in Salt Lake City, UT, paper 85-55 (March 1988).

51. G. Dixon-Lewis, Eighteenth Symposium (Int.) on Combustion, p. 385, The Combustion Institute, Pittsburgh, PA (1981).

52. R. K. Hanson and S. Saliman, in Combustion Chemistry, W. C. Gardiner, Jr., ed., Springer, New York, NY (1984).

53. J. E. Mertens, D. F. Davidson, and R. K. Hanson, unpubliahed results.

54. T. R. Roose, Ph.D. Thesis, Stanford University, Stanford, CA (1981). 